\title{
Avaliação ambiental, econômica e social de um novo compósito cimentício produzido com elevado teor fibra de coco tratada
}

A utilização de fibras vegetais pode trazer inúmeras vantagens, dentre elas, se destacam a obtenção de matérias-primas renováveis, baixo custo, produção sem poluição e oportunidade de desenvolvimento agrícola sustentável. Além disso, o reaproveitamento da fibra vegetal na construção civil pode tanto diminuir o consumo de agregados minerais como areia e brita, quanto melhorar propriedades físicas e mecânicas de novos compósitos. Nesse sentido, em busca de se obter um compósito cimentício não apenas de elevada resistência e durabilidade, mas também econômico e ecológico, no presente trabalho um novo compósito cimentício produzido com elevado teor de fibra de coco tratada foi avaliado utilizando os métodos Ambitec/Uesc para a questão socioeconômica e a Avaliação do Ciclo de Vida (ACV) para avaliação do desempenho ambiental. De acordo com os resultados obtidos, conclui-se que a combinação entre látex natural e sílica ativa proporciona uma solução econômica, social e ambientalmente adequada para o aproveitamento da fibra de coco na construção civil.

Palavras-chave: Sustentabilidade; Fibra Vegetal; Cimento Portland.

\section{Environmental, economic and social analysis of a new cement composite produced with high percentage of treated coconut}

The use of coconut fibers for the production of mortars and concretes can bring numerous benefits. It is a renewable material and high availability, and thus a lowcost production. Furthermore, the se of coconut fiber in civil building can both reduce the consumption of mineral aggregates such as sand and gravel as well as improve the physical and mechanical properties of the new composite. In this sense, seeking to obtain a cementitious composite not only high strength and durability, but also economic and ecological, in this study a new cement composite produced with high percentage of treated coconut was evaluated using the Ambitec/Uesc methods for socio-economic evaluation and Life-cycle Assessment (LCA) for its environmental performance. Based on the results obtained in this study, we can conclude that the combination of natural latex and silica fume provides an appropriate economic, social and environmental solution for the use of coconut fiber in civil building.

Keywords: Sustainability; Vegetable Fiber; Portland Cement

Topic: Engenharia da Sustentabilidade e Meio Ambiente

Reviewed anonymously in the process of blind peer
Received: $10 / 04 / 2018$

Approved: 24/05/2018
Everton José da Silva (iD

Instituto Federal da Bahia, Brasil

http://lattes.cnpq.br/9664150435542820

http://orcid.org/0000-0002-5653-5106

is everton@yahoo.com.br

Fermin de la Caridad Garcia Velasco (it)

Universidade Estadual de Santa Cruz, Brasi

http://lattes.cnpq.br/2300280094545118

http://orcid.org/0000-0003-0803-9148

fermingv@gmail.com

Francisco Heriberto Martínez Luzardo (iD)

Universidade Estadual de Santa Cruz, Brasil

http://lattes.cnpq.br/4333332503448953

http://orcid.org/0000-0003-1706-8231

fmartinezluzardo@gmail.com
Henrique Leonardo Maranduba (iD)

Universidade Estadual de Santa Cruz, Brasil

http://lattes.cnpq.br/7176196651714951

http://orcid.org/0000-0002-4358-5366

henrique.leo@gmail.com

Maria Lidiane Marques (iD

Instituto Federal da Bahia, Brasil

http://lattes.cnpq.br/0896370936952827

http://orcid.org/0000-0001-6837-3389

lidiane marques@yahoo.com.b
Referencing this:

SILVA, E. J.; VELASCO, F. C. G.; LUZARDO, F. H. M.; MARANDUBA, H. L.; MARQUES, M. L.. Avaliação ambiental, econômica e social de um novo compósito cimentício produzido com elevado teor fibra de coco tratada. Revista Ibero Americana de Ciências Ambientais, v.9, n.4, p.253-267, 2018. DOI: http://doi.org/10.6008/CBPC21796858.2018.004.0021 


\section{INTRODUÇÃO}

A atividade da construção civil é reconhecidamente importante nos aspectos sociais e econômicos pelas peculiaridades de sua cadeia produtiva: setor de mão de obra intensiva, baixo coeficiente de importação e elevados efeitos multiplicadores sobre a economia (renda, emprego e tributos). Teixeira et al. (2005) afirmam que $94 \%$ da indústria da construção civil brasileira é composta por micro e pequenas empresas que possuem baixo coeficiente técnico e empregam trabalhadores de baixa qualificação formal.

Entretanto, o grande desafio do setor é implementar um programa de desenvolvimento sustentável capaz de reduzir os impactos ambientais por ele causados. Nesse sentido, o setor construtivo tem muito a contribuir, principalmente no que concerne ao aproveitamento de resíduos, transformando-os em subprodutos com valor agregado. A este respeito, a resolução do CONAMA (Conselho Nacional do Meio Ambiente) de número 307 define, classifica e estabelece os possíveis destinos finais dos resíduos de construção e demolição, atribuindo responsabilidades para o poder municipal e também para os geradores de resíduos no que se refere à sua destinação.

De acordo com Batlouni Neto (2007), a melhor estratégia para atender a esta resolução passa a ser a de empregar materiais que gerem menos resíduos, ou aqueles que possam ser reutilizados ou reciclados, dentro ou fora do canteiro de obras, desde que no canteiro seja implantado um sistema de separação e disposição desses materiais (coleta seletiva).

Uma das possibilidades para o aproveitamento de resíduo pela construção civil é o emprego de fibras vegetais para produção de compósitos cimentícios. Nas últimas décadas, tem havido um enorme esforço da comunidade científica para utilizar fibras vegetais na construção civil de maneira simples, barata e eficiente. Contudo, nota-se que as pesquisas nesta área ficam 'limitadas' a investigar características técnicas e científicas do material como: propriedades físicas, mecânicas, durabilidade e microestrutura do compósito sem um enfoque específico em relação às questões ambientais, econômicas e sociais diretamente envolvidas no processo.

A destinação inadequada do resíduo do coco verde, por exemplo, pode gerar graves problemas ambientais e sociais. A proliferação de vetores, em razão do acúmulo de água nas cascas, pode servir de criadouro para insetos se procriarem e transmitirem doenças graves. Além disso, as cascas de coco demandam uma grande área nos aterros para a deposição devido a sua lenta degradação e difícil compactação em função da sua constituição fibrosa. A poluição visual causada pelo resíduo também desfavorece iniciativas para intervenções sociais e econômicas nos locais onde o descarte não é adequado. Por esse conjunto de fatores, torna-se importante quantificar os ganhos proporcionados pela reutilização de fibras vegetais na construção civil.

Existem algumas metodologias capazes de mensurar tecnicamente quais os benefícios que a incorporação de fibra vegetais em compósitos cimentícios pode promover em relação às questões associadas ao meio ambiental, econômico e social. No presente trabalho, foram utilizadas as ferramentas 'Avaliação do 
ciclo de vida' (ACV) (2006) e a AMBITEC/UESC (2014), uma adaptação da metodologia AMBITEC/EMBRAPA (2004).

De acordo com a International Organization for Standardization - ISO 14040, a ACV é a compilação e avaliação das entradas, das saídas e dos impactos ambientais potenciais de um sistema de produto ao longo do seu ciclo de vida. A metodologia de ACV é dividida em 4 fases interativas, a saber: definição de objetivo e escopo, análise de inventário, avaliação de impactos e interpretação de resultados.

Na primeira fase da ACV, deve-se declarar o motivo, razões, fronteiras do sistema, unidades funcionais e público alvo do estudo. Já na segunda fase coletam-se os dados e procedimentos de cálculo para quantificar as entradas e saídas pertinentes de um sistema de produto. Isso consiste em um balanço de energia e massa que configura o inventário de ciclo de vida (ICV). A terceira fase é dirigida à significância dos impactos ambientais potenciais, usando os resultados da análise de inventário do ciclo de vida. Na quarta fase as constatações da análise do inventário e da avaliação de impacto são realizadas e combinadas, de forma consistente, com o objetivo e o escopo definidos, visando alcançar conclusões e recomendações. Entre todas as fases deve haver uma interpretação sistêmica de todo o processo (ISO 14040, 2006).

Alguns trabalhos recentemente publicados têm utilizado a ACV para avaliar os materiais compósitos do ponto de vista ambiental. Borges et al. (2014) utilizaram esta ferramenta para comparar o impacto ambiental de $1 \mathrm{~m}^{3}$ produzido de concreto convencional (cimento Portland, areia, brita e água) com $1 \mathrm{~m}^{3}$ de concreto geopolimérico (o geopolímero é utilizado como aglomerante ao invés da pasta de cimento Portland). Os autores concluem que o concreto geopolimérico reduz o consumo energético em $45,8 \%$ e as emissões de $\mathrm{CO}_{2}$ em 72,4\% em comparação ao concreto tradicional.

O uso de fibras de polipropileno originais e recicladas para produção de concreto foi estudada por Yin et al. (2016) através da ACV. Todas as atividades necessárias para produzir os materiais foram consideradas, ou seja, fabricação, transporte, e, também, reciclagem/reprocessamento de resíduos. De acordo com os resultados, o concreto com fibras de polipropileno reciclado pode economizar $50 \%$ de $\mathrm{CO}_{2}$ equivalente, $65 \%$ de $\mathrm{PO}_{4}$ equivalente e $29 \%$ de água em relação ao concreto produzido com fibras originais.

Outros trabalhos como Duigou et al. (2014), Hervy et al. (2015) e llg et al. (2016) utilizaram a metodologia ACV para comparar materiais compósitos poliméricos tradicionais com compósitos reforçados com fibras naturais. Em todos os casos, foi constatada uma diminuição de impactos ambientais em função da reutilização de fibras vegetais e diminuição da quantidade de materiais originais necessários para produção do compósito.

A metodologia AMBITEC/UESC (2014), desenvolvida com base na metodologia AMBITEC/EMBRAPA [2004] trata-se método para avaliar impactos ambientais e sociais das introduções de alternativas tecnológicas como, por exemplo, a produção de compósitos cimentícios com fibras vegetais. Velasco (2013) realizou uma adequação da matriz de avaliação de impactos tecnológicos no método AMBITEC/UESC proposto. Esta nova metodologia contemplou a introdução de novos indicadores necessários para considerar adequadamente as características dos processos tecnológicos de reaproveitamento de resíduos e a caracterização social e ambiental. 
A Empresa brasileira de pesquisa agropecuária (EMBRAPA) desenvolveu o sistema de avaliação de impacto ambiental/social da inovação tecnológica (AMBITEC) para os setores: agropecuário, agroindustrial e de produção animal. Um elemento fundamental que conforma o sistema citado é a sua identificação com a avaliação dos impactos em todos os estágios dos processos sob avaliação, desde o planejamento e seleção do projeto até sua implementação (RODRIGUES et al., 2003). Esta metodologia constitui uma importante ferramenta que subsidia estudos de projetos ou programas de pesquisas relativas à implantação de tecnologias.

O sistema AMBITEC da EMBRAPA tem uma estrutura hierárquica simples, aplicado em escala local, e envolve a seleção de indicadores e seus componentes numa plataforma (matriz) operacional que inclui a medida ponderada dos pesos relativos de cada indicador. Sendo que os resultados do método são expressos tanto de forma integrada, em um índice geral de impactos, como de forma separada, por coeficientes de impactos e por indicadores (IRIAS et al., 2004).

Mediante uma análise de caso, Velasco (2013) identificou cada elemento que integra o AMBITEC/EMBRAPA e realizou uma avaliação analítica relativa aos indicadores e componentes que deveriam ser modificados, omitidos ou incorporados ao sistema de avaliação de impacto elaborado, conforme as especificidades das tecnologias, condições reais da localidade onde se aplicarão as tecnologias, e à disponibilidade das informações correspondentes ao objeto de estudo.

A autora descobriu que a questão do reaproveitamento de fibras vegetais para compósitos cimentícios não é adequadamente retratada nos indicadores do sistema, pelo que foi necessário, propor e realizar um conjunto de modificações (inclusões de novas variáveis) no sistema AMBITEC/EMBRAPA para elaboração do sistema AMBITEC/UESC.

A metodologia AMBITEC/UESC foi utilizada por Velasco et al. (2014) com o objetivo de avaliar os impactos socioambientais da implantação de novas tecnologias em agroindústrias, cuja matéria-prima fundamental são resíduos agroflorestais. A pesquisa colocou em foco inovações tecnológicas ligadas ao aproveitamento de resíduos de coco na produção de adsorventes naturais de metais em água e na produção de materiais de construção. De acordo com os autores, os resultados obtidos dos coeficientes de impacto e o Índice Geral de Impacto, nos âmbitos ambiental e social dos cenários estudados, indicam a viabilidade da implementação das novas tecnologias estudadas.

Dessa forma, com o objetivo avaliar (ambiental, econômica e socialmente) o compósito desenvolvido por Silva et al. (2018), ou seja, um novo compósito cimentício produzido com elevado teor de fibra de coco tratada, o presente trabalho utilizou as metodologias ACV e AMBITEC/UESC. Esta avaliação é de extrema importância, pois ela traz consigo garantias para o adequado aproveitamento da fibra de coco na construção civil.

\section{MATERIAIS E MÉTODOS}

\section{Avaliação do Ciclo de Vida}


Para avaliar o desempenho ambiental do compósito desenvolvido por Silva et al. (2018), utilizou-se a Avaliação do Ciclo de Vida (ACV). Xu et al. (2008) recomendam esta avaliação pois o uso de fibras naturais em materiais compósitos não os transformam, automaticamente, em compósitos sustentáveis, ou seja, a utilização das fibras em compósitos pode não ser, necessariamente, ambientalmente adequada.

A partir disso, foram considerados três tipos de compósitos: sem fibras de coco (S-F), com fibras de coco naturais (F-N) e com fibras de coco tratadas com látex e pozolana sílica ativa (S-A), conforme o trabalho realizado por Silva et al. (2018). Com essas informações foi possível fazer uma análise ambiental comparativa entre a produção de cada compósito. Conforme mostrado nos fluxogramas da Figuras 1, 2 e 3, considerouse a abordagem berço-portão, onde apenas os insumos foram considerados (as energias utilizadas no processo de produção foram desconsideradas pois a produção dos compósitos foi manual).

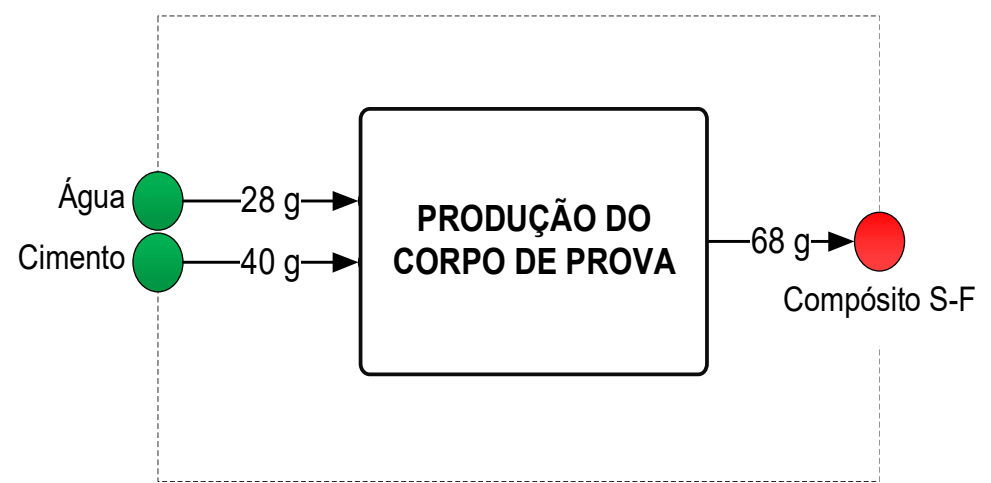

Figura 1: Fluxograma do sistema de produto - produção do compósito S-F.

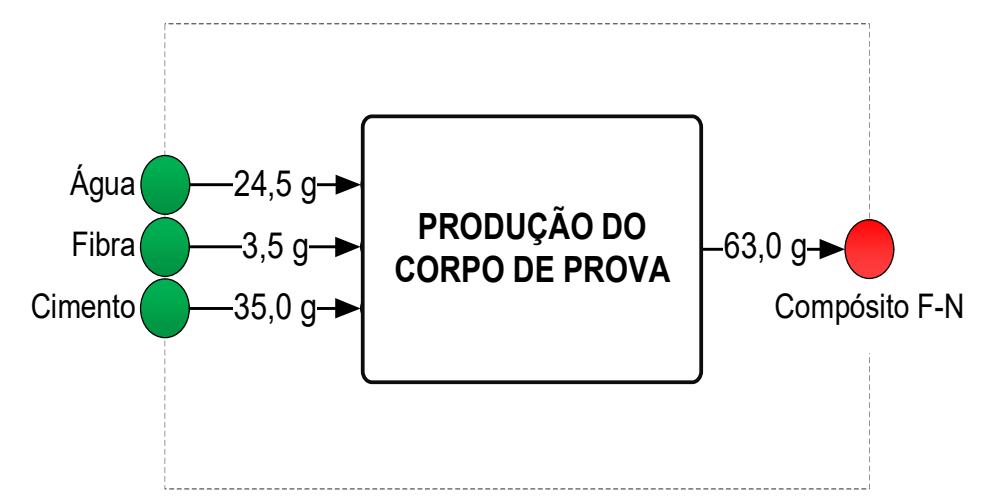

Figura 2: Fluxograma do sistema de produto - produção do compósito F-N.

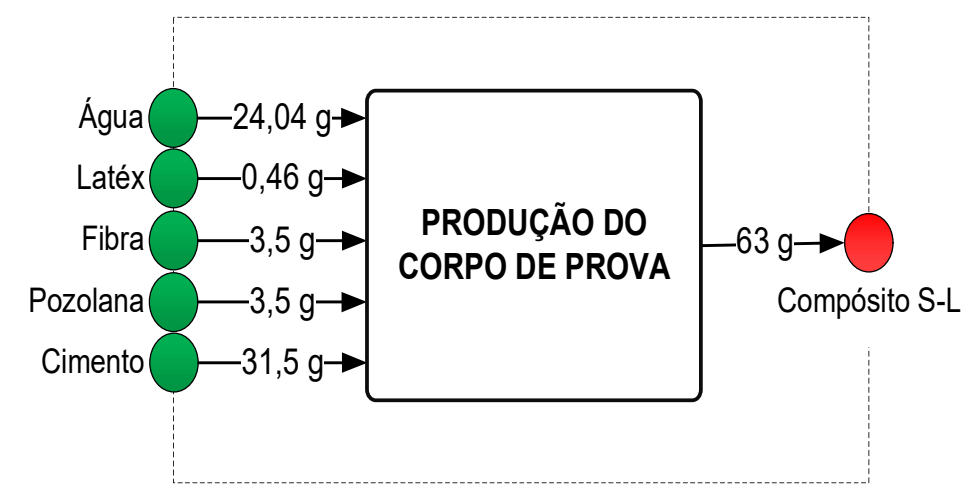

Figura 3: Fluxograma do sistema de produto - produção do compósito S-L.

Todos os inventários do ciclo de vida (ICV) dos insumos utilizados no processo de produção de cada compósito foram retirados da base de dados ecoinvent 3.01, e os cálculos dos impactos no ciclo de vida 
foram realizados por meio do software proprietário Simapro ${ }^{\circledast}$ 8.4.0.0. Os dados inseridos no SimaPro 8.1 para descrição do processo de descascamento do coco e geração das fibras foram obtidos a partir do trabalho de Bitencourt (2008). Para a água para usada na produção dos compósitos foi selecionada a opção da base de dados ecoinvent 3.01 'Tap water, at user (RoW)_tap water production and supply_Alloc Def U'. Para o cimento Portland foi selecionado o material 'Cement, Portland (RoW) Market for Alloc Def U'. Para o látex natural foi selecionado o material 'Latex (RoW) production Alloc Def U'. E para a pozolana sílica ativa foi selecionado o processo 'Hard coal ash (RoW) cement production, pozzolana and fly ash 36-55\%, non-US Alloc Def, $U^{\prime}$.

As categorias de impacto relevantes analisadas estão contidas no método ReCiPe (Midpoint H/V1.08/ World Recipe/Caracterização), sendo elas: Mudanças Climáticas; Acidificação Terrestre; Eutrofização de Água Doce; Eutrofização Marinha; Formação de Oxidante Fotoquímico; Formação de Particulados; Ocupação de Área Urbana; Depleção Hídrica e Depleção Fóssil.

Com relação ao transporte (rodoviário), foram consideradas as distâncias de $500 \mathrm{~km}$ para o cimento Portland e $1500 \mathrm{~km}$ para a pozolana sílica ativa, referentes aos seus respectivos locais de produção e a cidade de llhéus-BA. A distância de distribuição do látex foi desconsiderada pois este material é produzido no próprio município. Uma análise de incerteza através do método Monte Carlo em associação com matriz pedigree com intervalo de confiança de $95 \%$ e 1000 iterações foi realizada para comparar os desempenhos ambientais da produção dos compósitos. O vetor Pedigree utilizado nos inventários foi o $(1,5,1,1,5)$ com variância de 2,05 .

\section{AMBITEC/UESC}

A metodologia utilizada para avaliar o novo compósito cimentício do ponto de vista ambiental e social foi o método AMBITEC - EMBRAPA, adaptado por Velasco (2013). O sistema AMBITEC/UESC é direcionado a avaliar os impactos sócio-econômicos e ambientais das inovações tecnológicas no âmbito agrícola considerando o reaproveitamento de resíduos. Este método possui uma estrutura hierárquica simples, aplicado em escala local, e envolve a seleção de indicadores e seus componentes numa plataforma (matriz) operacional, que inclui a medida ponderada dos pesos relativos de cada indicador.

No caso dos indicadores da dimensão ambiental, as variáveis estão associadas aos efeitos correspondentes ao uso dos recursos naturais e à degradação do meio ambiente, com a introdução tecnológica. As principais questões abordadas aparecem organizadas nos seguintes indicadores: Uso de insumos químicos e materiais; Uso de energia; Uso de recursos naturais; Atmosfera; Geração de resíduos sólidos; Qualidade da água; RRR (Redução, reutilização e reciclagem) e Qualidade do produto.

$\mathrm{Na}$ dimensão dos indicadores relativos aos impactos sociais, consideraram-se aqueles que caracterizaram de alguma maneira as possíveis mudanças causadas pela introdução tecnológica sobre o modo de vida, a saúde, o bem-estar e a qualidade de vida dos moradores da comunidade. Tais indicadores correspondentes aos principais elementos relacionados aos impactos sociais foram: Capacitação; Oportunidade de emprego local qualificado; Oferta de emprego e condição do trabalhador; Qualidade de 
emprego; Geração de renda; Valor da propriedade; Saúde ambiental e social; Dedicação e perfil do responsável; Condição de comercialização; Reciclagem de resíduos e Relacionamento institucional.

Além das modificações acima, referentes à inclusão de novos indicadores (e a não aplicação de alguns presentes no AMBITEC/EMBRAPA), o AMBITEC/UESC utilizado no presente trabalho manteve toda a sua estrutura de cálculo similar ao AMBITEC/EMBRAPA, ou seja, foram utilizadas para os cálculos dos coeficientes de impacto e do índice geral de impacto as fórmulas, tal como aparecem reportadas na metodologia original. Este sistema, em essência, obtém um índice integrado de impactos composto de uma soma ponderada de indicadores que, por sua vez, é uma soma ponderada de componentes. No cálculo de cada indicador são considerados: o fator de ponderação da importância do componente (Pij); o coeficiente de alteração do componente (Aij); e o fator de escala de ocorrência espacial (Eij).

Conforme Velasco et al. (2014), os pesos dos indicadores foram determinados a partir da relação entre um valor unitário e a quantidade de indicadores. Para a determinação dos coeficientes de impacto, levou-se em consideração a equação 1 :

$$
C_{i}^{i m p}=\sum_{j=1}^{m} \text { Aij. Eij. Pij }
$$

Onde:

Ci imp.: coeficiente de impacto do indicador $\mathrm{i}$; Aij: coeficiente de alteração do componente j do indicador $i$; Eij: fator de ponderação para escala de ocorrência espacial do componente j do indicador i; Pij: fator de ponderação (K) para importância do componente j na composição do indicador i. $\mathrm{m}$ : número de componentes do indicador i.

Já o Índice Geral de Impacto da Inovação Tecnológica no âmbito ambiental ou social é obtido pela equação 2 .

$$
\mathrm{I}_{\text {tecn }}^{\mathrm{imp}}=\sum_{i=1}^{m} C_{i}^{i m p} \cdot P_{i}
$$

Onde:

limp tecn.: índice de impacto da tecnologia

Ci imp.: coeficiente de impacto do indicador $\mathrm{i}$

Pi: fator de ponderação para importância do indicador i para composição do índice de impacto da tecnologia. $\mathrm{m}$ : número de indicadores

Dessa forma, a nova tecnologia de incorporação de fibras vegetais em compósitos cimentícios é avaliada de forma integral pela composição do Índice de Impacto Ambiental e Social respectivamente, considerando o peso que the é conferido ao indicador de acordo com sua importância relativa na composição do impacto final da tecnologia.

É importante ressaltar que o beneficiamento do coco gera três tipos de resíduos a saber: pó de coco (fração inferior a $10 \mathrm{~mm}$ ) utilizado como substrato para a agricultura; fibra longa (fração superior a $30 \mathrm{~mm}$ ) utilizado para a indústria de cordas, estofamentos, vasos e outras aplicações; e a fibra curta (fração intermediária entre 10 e $30 \mathrm{~mm}$ ) ainda sem uma destinação específica, por isso, a fibra curta com 
comprimento médio de $25 \mathrm{~mm}$ foi adotada para a produção do compósito cimentício elaborado por Silva et al. (2018).

A Figura 4 exibe o fluxograma operacional do processo tecnológico para a produção de tijolos maciços utilizando o compósito cimentício com elevado teor de fibra de coco tratada (mistura S-L). Este fluxograma foi utilizado para balizar a utilização da metodologia AMBITEC/UESC através de dois cenários: produção de um tijolo com a tecnologia convencional e com a tecnologia de reaproveitamento da fibra de coco nas condições propostas no presente trabalho, a partir do resíduo gerado durante o beneficiamento do coco para a produção e comercialização da água de coco.

\section{Análise econômica}

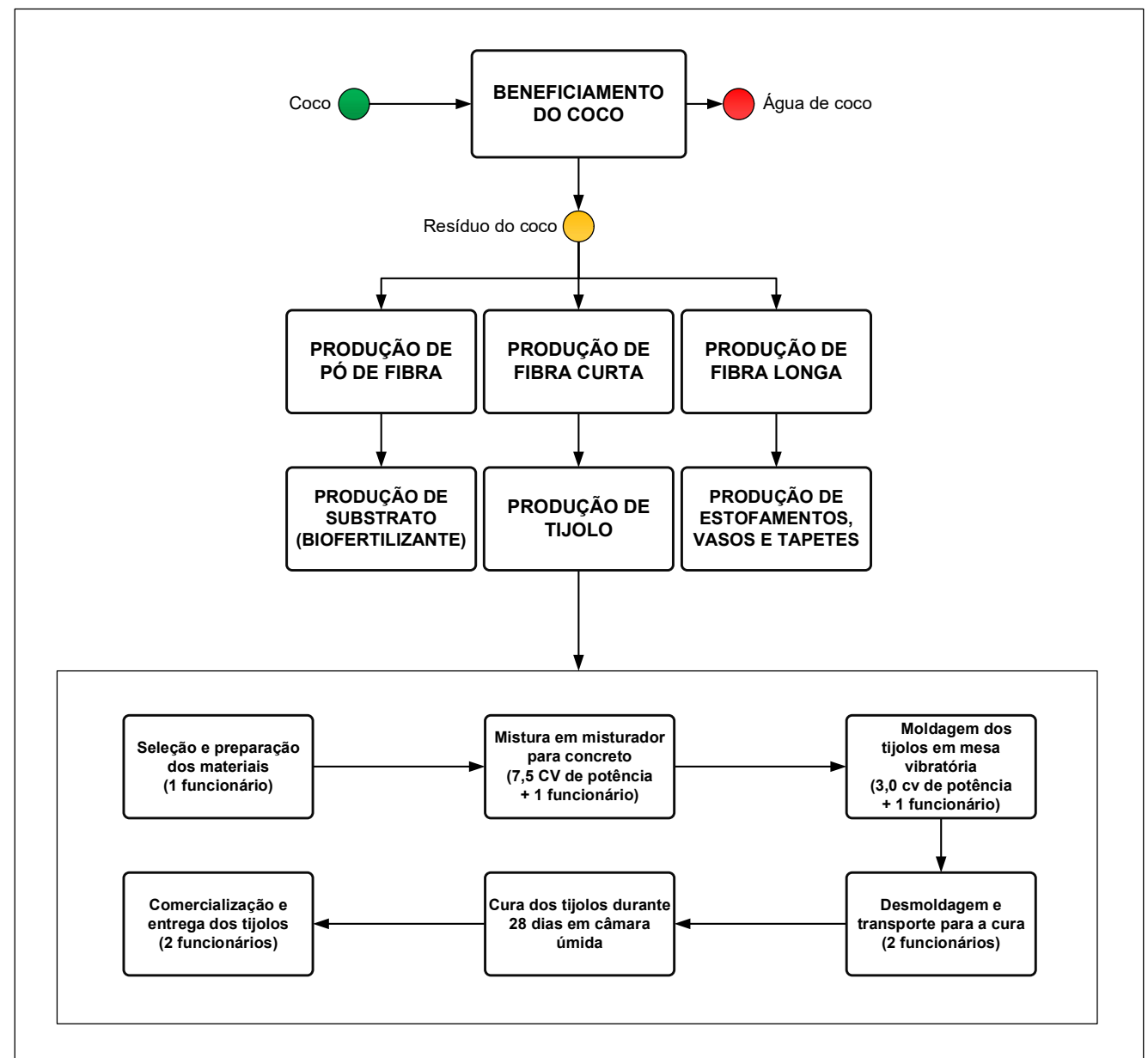

Figura 4: Fluxograma de produção do tijolo maciço com elevado teor de fibra de coco tratada para análise através da AMBITEC/UESC.

Para avaliar a questão econômica/financeira da utilização desta tecnologia para a produção de compósitos cimentícios com fibras vegetais, optou-se como metodologia de cálculo a comparação entre os custos dos materiais unitários para a produção de um tijolo maciço e suas respectivas quantidades necessárias para produzir uma unidade. Para tanto, o SINAPI (Sistema Nacional de Pesquisa de Custos e Índices da Construção Civil 2016) foi utilizado como fonte para os valores unitários do cimento Portland e a pozolana sílica ativa. A EMBASA (Empresa Baiana de Águas e Saneamento) foi utilizada como valor de referência para a água da rede de distribuição e o CIF (Centro de Inteligência em Florestas) como referência 
para o valor do látex natural. Após realizar o somatório entre as quantidades de materiais necessárias para cada tijolo multiplicadas por seus respectivos valores unitários, foi possível estabelecer o valor total necessário para produção individual de cada tijolo.

Duas ponderações são importantes nesta avaliação. A primeira é a não contabilização do custo para a geração da fibra de coco. Isso se deve ao fato de a fibra ser um resíduo agroindustrial sem destinação, ou seja, sua não utilização se tornaria um passivo ambiental, por isso, eventuais custos para sua geração não foram considerados nesta análise. Outro ponto é o sistema de produção mecânica dos tijolos maciços, considerou-se uma equivalência de custos com energia para a produção dos tijolos maciços (com e sem a presença das fibras) nesta avaliação.

\section{RESULTADOS E DISCUSSÃO}

\section{Avaliação do Ciclo de Vida}

A Figura 5 apresenta o desempenho ambiental dos compósitos efetuado a partir de uma avaliação do ciclo de vida que comparou os materiais e suas quantidades utilizados para a produção de cada compósito. Nota-se que o compósito sem incorporação da fibra de coco (S-F) aparece como maior impacto ambiental em quase todas as categorias avaliadas, com exceção da categoria Depleção fóssil onde o compósito com fibras tratadas com látex natural e pozolana sílica ativa (S-L) predominou como o cenário de maior impacto. Por outro lado, o compósito com fibras naturais de coco sem tratamento (F-N) apresentou-se como aquele com o melhor desempenho ambiental, com uma média de impacto $10 \%$ inferior à S-F, na maioria das categorias. Isso pode ser atribuído ao uso da fibra de coco como componente substituto do cimento, grande causador de impacto ambiental (BOESCH et al., 2010).

Em relação ao compósito com fibras tratadas com látex natural e pozolana sílica ativa (S-L), também há uma importante redução de impactos em relação ao compósito S-F. Algumas categorias como depleção hídrica, mudança climática e eutrofização da água doce apresentaram impactos menores que a produção do compósito F-N, possivelmente devido a menor quantidade de cimento Portland utilizada na confecção do compósito S-L em relação ao F-N.

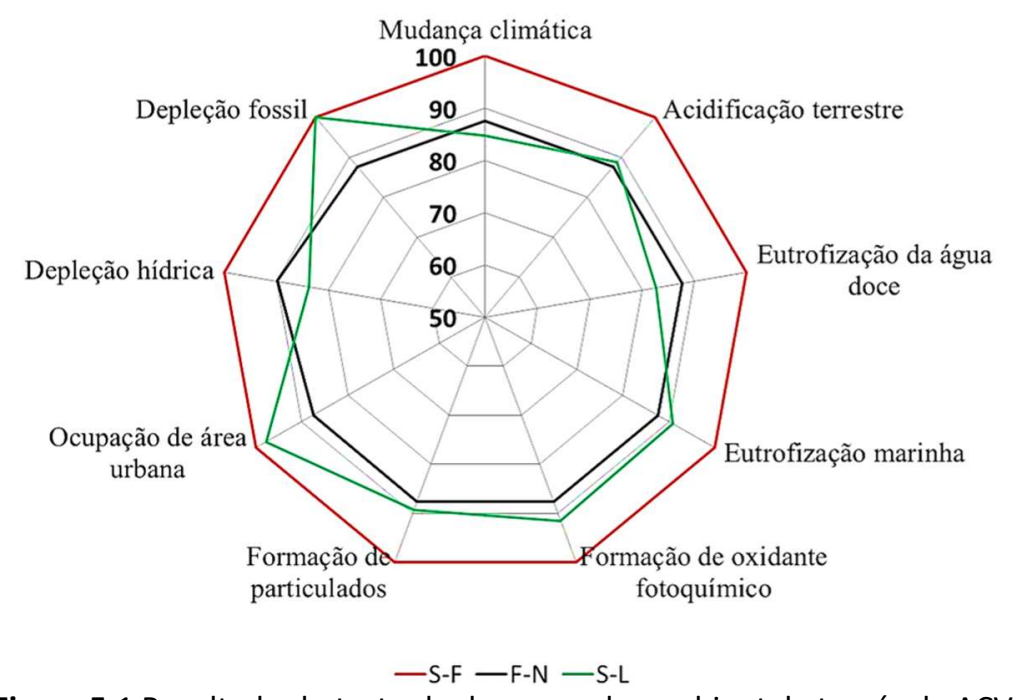

Figura 5:1 Resultado do teste de desempenho ambiental através da ACV. 
Já as categorias ocupação de área urbana e depleção fóssil apresentaram valores próximos ao compósito S-F. Isso pode ser atribuído à utilização de látex natural e a grande distância de transporte considerada para a pozolana sílica ativa, $1500 \mathrm{~km}$. Marques (2014) e Christoforou et al. (2015) afirmam que o transporte é uma variável importante no desempenho ambiental de um produto do setor construtivo.

Para avaliar as incertezas inerentes aos dados do inventário do ciclo de vida, bem como a escala de produção dos compósitos, foi realizada uma análise de incerteza, utilizando o método iterativo de Monte Carlo, considerando 1000 iterações e um intervalo de confiança de 95\%. Os resultados dessa análise permitem afirmar com $100 \%$ de confiança que, em relação ao compósito S-F, o F-N apresentou impacto ambiental menor em todas as categorias avaliadas. Ainda em relação ao compósito S-F, o S-L apresentou redução de impacto ambiental em seis categorias (depleção hídrica, ocupação de área urbana, formação de particulados, eutrofização da água doce, acidificação terrestre e mudança climática) em um total de nove avaliadas. Em outras palavras, há uma diminuição de impactos ambientais quando se utiliza a fibra de coco natural ou com o tratamento proposto por Silva et al. (2018).

Ao se realizar a análise comparativa entre os compósitos F-N e S-L, pode-se afirmar com $100 \%$ de confiança que o compósito S-L reduz os impactos ambientais nas categorias depleção hídrica, ocupação de área urbana, eutrofização da água doce e mudança climática, por outro lado, aumenta os impactos ambientais na categoria depleção fóssil. Não há conclusões claras em relação às categorias formação de particulados, formação de oxidante fotoquímico, eutrofização marinha e acidificação terrestre. Neste contexto, pode-se considerar que há uma redução de impactos ambientais do compósito S-L em relação ao F-N.

Ressalta-se que o resultado da avaliação comparativa entre o ciclo de vida do compósitos S-L e S-F está de acordo com Bribián et al. (2011). Esses autores demonstram que o impacto dos produtos de construção civil pode ser significativamente reduzido através da promoção da utilização das melhores técnicas disponíveis e eco-inovação em plantas de produção. Substitui-se o uso de recursos naturais finitos por resíduos gerados em outros processos de produção, de preferência disponíveis localmente, como o caso fibras de coco. Isto estimularia a concorrência entre os fabricantes a lançar mais produtos eco-eficientes e incentivaria a utilização das declarações de produtos ambientais.

A partir do exposto, pode-se afirmar que o tratamento utilizando a combinação entre látex natural e a pozolana sílica ativa apresentou um adequado desempenho ambiental através da diminuição dos impactos causados em função da menor quantidade de cimento Portland utilizada. Constata-se que a utilização da fibra de coco nestas condições, para a produção de argamassas e concretos, pode atender a resolução do CONAMA de número 307/2002.

\section{AMBITEC/UESC}

As Tabelas 1 e 2 apresentam os resultados obtidos a partir da aplicação da metodologia AMBITEC/UESC considerando o cenário da produção de tijolos sem a incorporação da tecnologia do tratamento protetor das fibras (tijolo com a tecnologia -convencional) e o cenário com a utilização do 
tratamento protetor para as fibras de coco inseridas nos tijolos (tijolo com a tecnologia de reaproveitamento da fibra).

Tabela 1: Índice Geral de Impacto Ambiental nos casos estudados segundo a metodologia AMBITEC/UESC.

\begin{tabular}{|c|c|c|c|c|c|c|}
\hline \multirow[t]{2}{*}{$\begin{array}{l}\text { Indicadores de Impacto } \\
\text { Ambiental }\end{array}$} & \multicolumn{3}{|c|}{ Tijolo com a tecnologia convencional } & \multicolumn{3}{|c|}{$\begin{array}{l}\text { Tijolo com a tecnologia de reaproveitamento da } \\
\text { fibra }\end{array}$} \\
\hline & $\begin{array}{ll}\text { Peso } & \text { do } \\
\text { Indicador }\end{array}$ & $\begin{array}{l}\text { Coeficiente de } \\
\text { Impacto }\end{array}$ & $\mathrm{Ci} \times \mathrm{Pi}$ & $\begin{array}{ll}\text { Peso do } \\
\text { Indicador }\end{array}$ & $\begin{array}{l}\text { Coeficiente de } \\
\text { Impacto }\end{array}$ & $\mathrm{Ci} \times \mathrm{Pi}$ \\
\hline $\begin{array}{l}\text { Uso de insumos químicos e } \\
\text { materiais }\end{array}$ & 0,125 & -1 & $-0,125$ & 0,125 & $-0,5$ & $-0,0625$ \\
\hline Uso de Energia & 0,125 & $-1,5$ & $\overline{-}-1875$ & 0,125 & -1 & $-0,125$ \\
\hline Uso de recursos naturais & 0,125 & -2 & $-0,25$ & 0,125 & -1 & $-0,125$ \\
\hline Afmosfera & 0,125 & $-0,5$ & 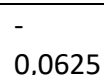 & 0,125 & $-0,1$ & $-0,0125$ \\
\hline $\begin{array}{l}\text { Geração de resíduos } \\
\text { sólidos }\end{array}$ & 0,125 & $-0,5$ & $\overline{-}, 0625$ & 0,125 & $-0,25$ & $\overline{-}, 03125$ \\
\hline Qualidade de água & 0,125 & $-0,5$ & $\overline{-}-0625$ & 0,125 & $-0,5$ & $-0,0625$ \\
\hline RRR & 0,125 & 0 & 0 & 0,125 & 5 & 0,625 \\
\hline Qualidade do produto & 0,125 & 0 & 0 & 0,125 & 4,5 & 0,5625 \\
\hline Valor total & 1 & & $-0,75$ & 1 & & 0,76875 \\
\hline $\begin{array}{l}\text { Índice Geral de Impacto } \\
\text { Ambiental }\end{array}$ & \multicolumn{3}{|l|}{$-0,750$} & \multicolumn{3}{|l|}{0,769} \\
\hline
\end{tabular}

Observa-se que o índice geral de impacto ambiental foi negativo $(-0,750)$ para a produção de tijolo sem a tecnologia de reaproveitamento e positivo $(0,769)$ para a produção do tijolo com a tecnologia. Isso pode ser atribuído a menor quantidade de cimento Portland e água necessários para produção do tijolo com a presença da fibra de coco tratada. Este tijolo utiliza 21,25\% menos cimento Portland e 14,04\% menos água. Embora ele consuma mais energia para ser produzido, conforme constatado pelo coeficiente de impacto do indicador "uso de energia", há uma compensação pelo coeficiente de impacto do indicador RRR (Redução, Reutilização e Reciclagem). Isso influiu significativamente pelo fato dele potencializar o gerenciamento de resíduos na entrada de insumos do sistema (VELASCO et al., 2014). Dessa forma, esta nova tecnologia pode minimizar o volume de resíduos sólidos provenientes de atividades industriais do processamento de coco.

Tabela 2: Índice Geral de Impacto Social nos casos estudados segundo a metodologia AMBTEC/UESC.

\begin{tabular}{|c|c|c|c|c|c|c|}
\hline \multirow[t]{2}{*}{ Indicadores de Impacto Social } & \multicolumn{3}{|c|}{ Tijolo com a tecnologia convencional } & \multicolumn{3}{|c|}{$\begin{array}{l}\text { Tijolo com a tecnologia de reaproveitamento } \\
\text { da fibra }\end{array}$} \\
\hline & $\begin{array}{l}\text { Peso do } \\
\text { Indicador }\end{array}$ & $\begin{array}{l}\text { Coeficiente de } \\
\text { Impacto }\end{array}$ & $\mathrm{Ci} \times \mathbf{P i}$ & $\begin{array}{l}\text { Peso do } \\
\text { Indicador }\end{array}$ & $\begin{array}{l}\text { Coeficiente de } \\
\text { Impacto }\end{array}$ & $\mathrm{Ci} \times \mathbf{P i}$ \\
\hline Capacitação & 0,083 & 0 & 0 & 0,083 & 0,35 & 0,02905 \\
\hline $\begin{array}{l}\text { Oportunidade de emprego } \\
\text { local qualificado }\end{array}$ & 0,083 & 0 & 0 & 0,083 & 0,75 & 0,06225 \\
\hline $\begin{array}{l}\text { Oferta de emprego e condição } \\
\text { do trabalhador }\end{array}$ & 0,083 & 0 & 0 & 0,083 & 0,7 & 0,0581 \\
\hline Qualidade de emprego & 0,083 & 0 & 0 & 0,083 & 0,45 & 0,03735 \\
\hline Geração de renda & 0,083 & 0 & 0 & 0,083 & 2,5 & 0,2075 \\
\hline Valor da propriedade & 0,083 & 0 & 0 & 0,083 & 2,5 & 0,2075 \\
\hline Saúde ambiental e social & 0,083 & -4 & $-0,332$ & 0,083 & -1 & $-0,083$ \\
\hline Segurança e saúde ocupacional & 0,083 & $-0,15$ & $-0,0125$ & 0,083 & $-0,45$ & $\begin{array}{l}- \\
0,03735\end{array}$ \\
\hline $\begin{array}{l}\begin{array}{l}\text { Dedicação e perfil do } \\
\text { responsável }\end{array} \\
\end{array}$ & 0,083 & 1,7 & 0,1411 & 0,083 & 1,7 & 0,1411 \\
\hline Condição de comercialização & 0,083 & 2,25 & 0,18675 & 0,083 & 2,7 & 0,2241 \\
\hline Reciclagem de resíduos & 0,083 & 0,4 & 0,0332 & 0,083 & 2 & 0,166 \\
\hline Relacionamento institucional & 0,083 & 2 & 0,166 & 0,083 & 2,5 & 0,2075 \\
\hline
\end{tabular}




\begin{tabular}{|l|l|l|l|l|l|}
\hline Valor total & 1 & $\mathbf{0 , 1 8 2 6}$ & 1 & 1,2201 \\
\hline Índice geral de impacto social & $\mathbf{0 , 1 8 3}$ & $\mathbf{1 , 2 2}$ \\
\hline
\end{tabular}

Em relação ao índice geral de impacto social, verificam-se valores positivos nos dois cenários, porém, consideravelmente maior em favor da produção de tijolo com a tecnologia de reaproveitamento. Isso pode ser explicado pelas novas oportunidades de emprego, a geração de renda e a possibilidade de novas produções que atenderiam perspectivas de mercado com um possível aumento da comercialização. Esses fatores estão presentes nos coeficientes de impacto dos indicadores: capacitação, oportunidade de emprego local qualificado, oferta de emprego e condição do trabalhador, qualidade do emprego, geração de renda e valor da propriedade.

Outro indicador que também percebeu o uso da tecnologia de reaproveitamento foi saúde ambiental e social. A diferença entre os cenários é atribuída à redução tanto da destinação inadequada de resíduos sólidos quanto da produção de cimento Portland. Adiciona-se a este argumento o fato de que o uso desta tecnologia para produção de tijolos constitui uma via para atribuir alto valor agregado a um resíduo que está sendo descartado inadequadamente. Dessa maneira, estes resíduos serão transformados em renda adicional para os estabelecimentos agroindustriais dedicados à atividade da produção de água de coco. Aos benefícios ambientais e sociais já apresentados, soma-se outro fator importante a respeito da incorporação de fibras vegetais em compósitos cimentícios: a redução de emissão de $\mathrm{CO}_{2}$.

A emissão de $\mathrm{CO}_{2}$ no Brasil causada pela produção de cimento Portland é da ordem de $659 \mathrm{kgCO} / \mathrm{t}$ (LIMA, 2010). A partir deste dado e considerando as 70 milhões de toneladas fabricadas em 2013, o Brasil produziu cerca de 46,13 milhões de toneladas de $\mathrm{CO}_{2}$ em 2013 devido a utilização de cimento Portland. A partir do teor de substituição de cimento Portland por fibra de coco proposto por Silva et al. (2018), para a produção de um mesmo volume de compósito, pode-se inferir que haveria uma redução do uso de cimento para aproximadamente 55 milhões de toneladas anuais. A "economia" de 15 milhões de toneladas de cimento geraria uma diminuição aproximada de 9,8 milhões de toneladas de $\mathrm{CO}_{2}$ lançadas na atmosfera anualmente.

Assim sendo, a utilização de fibras vegetais na produção de argamassas e concretos poderá contribuir significativamente para o uso e reuso racional de recursos naturais, reciclagem, eficiência energética e diminuição tanto da geração de resíduos quanto de gases do efeito estufa. Isto, por sua vez, implicará em aumento da qualidade de vida/bem-estar, aumento da formalidade dos empregos, qualificação e competitividade do setor construtivo nacional.

\section{Avaliação econômica}

Outro aspecto fundamental para a utilização das fibras de coco no setor construtivo está associado à questão econômica. A este respeito, a Tabela 3 apresenta os valores dos materiais utilizados para a produção dos tijolos utilizando as fibras de coco nas condições propostas.

Observa-se que o tijolo F-N reduziu o custo em $12,5 \%$ comparado ao S-F em função da diminuição da água e, principalmente, da redução do cimento Portland necessário para a produção de um mesmo volume 
de compósito. Entretanto, diante das condições de degradabilidade da fibra de coco inserida em compósitos cimetícios, torna-se necessário a utilização do tratamento para se garantir as condições adequadas de uso do material a longo prazo. Embora o tijolo S-L tenha registrado um pequeno aumento de 6,3\% em relação ao S-F, pode-se afirmar que o tratamento proposto por Silva et al. (2018) é competitivo do ponto de vista econômico diante de todos os benefícios técnicos, ambientais e sociais do novo compósito.

Tabela 3: Valores totais (R\$) para a produção dos tijolos maciços.

\begin{tabular}{|l|l|l|l|l|l|}
\hline \multirow{2}{*}{ Materiais utilizados } & \multirow{2}{*}{ Valor unitário (R\$/g) } & \multirow{2}{*}{ Fonte } & \multicolumn{3}{|l|}{ Quantidades de materiais para cada tijolo (g) } \\
\cline { 4 - 6 } & & & S-F & F-N & S-L \\
\hline Cimento Portland & 0,00058 & SINAPI & 960 & 840 & 756 \\
\hline Água & 0,00000642 & EMBASA & 672 & 588 & 576,96 \\
\hline Fibra de coco & $*$ & & 0 & 84 & 84 \\
\hline Látex Natural & 0,00207 & CIF & 0 & 0 & 11,04 \\
\hline Sílica Ativa & 0,00188 & SINAPI & 0 & 0 & 84 \\
\hline Valor total para cada tijolo produzido (R\$) & & $\mathbf{0 , 5 6 1 1}$ & $\mathbf{0 , 4 9 0 9}$ & $\mathbf{0 , 5 9 6 4}$ \\
\hline
\end{tabular}

* Valor desconsiderado por se tratar de um resíduo agroindustrial

Ainda em relação ao uso de pozolanas, a utilização de outras pozolanas oriundas de resíduos de biomassa produzidos localmente como cinzas de casca de arroz, cinza de bagaço de cana e resíduos da biomassa de cacau para a produção do compósito cimentício (SILVA et al., 2014; CORDEIRO, 2006, SILVA et al., 2015) poderiam diminuir ainda mais o valor necessário para a produção do novo compósito. Uma vez que se desconsidere o valor relativo à pozolana, o custo do tijolo com fibra de coco tratada (Figura 6) seria de R\$ 0,4385 , ou seja, $21,85 \%$ mais barato que um tijolo composto por cimento Portland e água.

Para uma análise comparativa com um produto similar do mercado, de acordo com o índice da SINAPI (dezembro de 2015) o valor unitário de um tijolo maciço cerâmico aparente (Figura 7) custava $R \$$ 0,87, ou seja, praticamente o dobro do valor de um tijolo (com mesmas dimensões) produzido com a formulação utilizando fibra de coco e cinza da biomassa de cacau, por exemplo. Além disso, deve-se considerar a escala de produção a nível laboratorial utilizada; espera-se obter custos de produção ainda mais vantajosos ao se adotar uma produção em escala industrial.

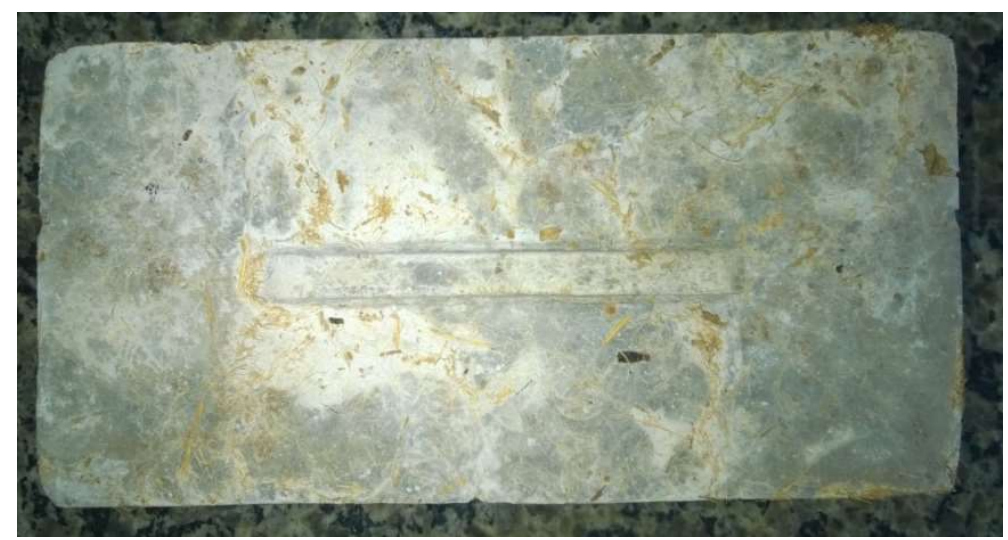

Figura 62: Novo tijolo maciço produzido com elevado teor de fibra de coco tratada. 


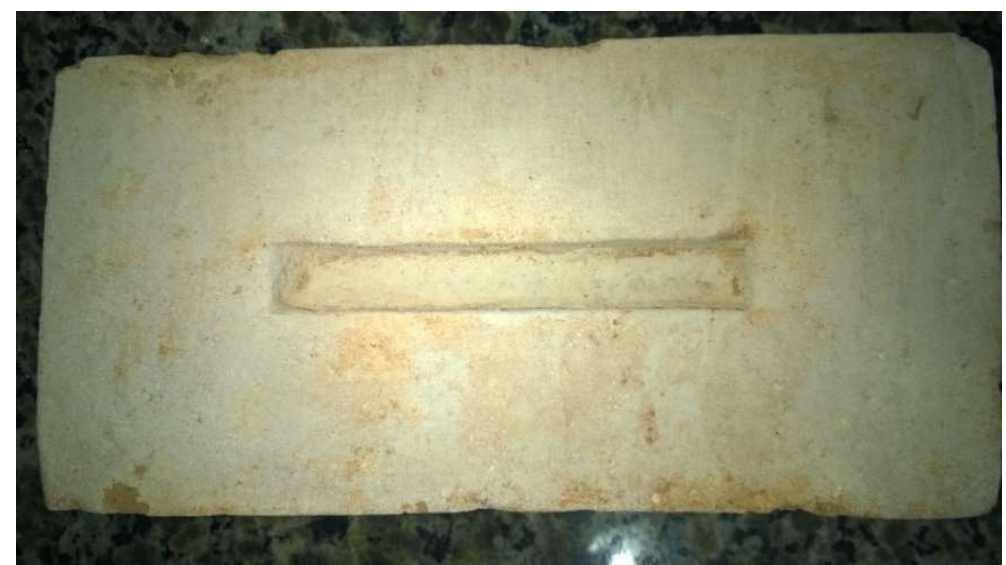

Figura 73: Tijolo cerâmico maciço aparente.

\section{CONCLUSÕES}

A partir dos resultados obtidos no presente trabalho, pode-se concluir através da avaliação ambiental, econômica e social que o novo compósito cimentício com elevado teor de fibra de coco tratada pode: diminuir impactos ambientais; gerar emprego e distribuição de renda para a comunidade; diminuir recursos naturais que seriam utilizados em materiais convencionais; e sua produção gerar uma economia nos insumos utilizados de até $21 \%$ em relação ao compósito cimentício convencional sem fibras.

As metodologias utilizadas no presente trabalho mostraram convergência dos indicadores ambientais da AMBITEC/UESC com categorias de impacto da ACV. Na metodologia ACV o novo compósito promoveu redução de impactos nas seguintes categorias: mudança climática, acidificação terrestre, eutrofização, e formações de oxidante fotoquímico e particulados. De forma congruente, na metodologia AMBITEC/UESC o novo compósito obteve melhor desempenho nos indicadores: uso de insumos, recursos naturais e energia, geração de resíduos, atmosfera, RRR e qualidade do produto.

Diante da boa relação custo/benefício ambiental-econômico-social, conclui-se que o uso do novo compósito obtido para construção de habitações de interesse social diminuiria impactos ambientais, reduziria o déficit habitacional existente, geraria emprego formal, renda e qualificação para a comunidade. Através disso, a construção civil brasileira poderá melhorar sua sustentabilidade com uso e reuso racional de recursos naturais, eficiência energética e diminuição dos gases do efeito estufa.

\section{REFERÊNCIAS}

BATLOUNI NETO, J.. Critérios de projetos para seleção de materiais. In: ISAIA, G. C.. Materiais de construção civil e princípios de ciência e engenharia de materiais. São Paulo: IBRACON, 2007. p.1792.

BITENCOURT, D. V.. Potencialidades e estratégias sustentáveis para o aproveitamento de rejeitos de coco (cocus nucifera). Dissertação (Mestrado em desenvolvimento e meio ambiente) - Universidade Federal de Sergipe, Aracaju, 2008.

BOESCH, M. E.; HELLWEG, S.. Identifying Improvement Potentials in Cement Production with Life Cycle Assessment. Environmental Science \& Technology, v.44, n.23, p.91439149, 2010. DOI: http://doi.org/10.1021/es100771k
BORGES, P. H. R.; LOURENÇO, T. M. F.; FOUREAUX, A. F. S.; PACHECO, L. S.. Estudo comparativo da análise de ciclo de vida de concretos geopoliméricos e de concretos a base de cimento Portland composto (CPII). Ambiente Construído, v.14, n.2, p.153-168, 2014.

BRIBIÁN, I. Z.; CAPILLA, A. V.; USÓN, A. A.. Life cycle assessment of building materials: Comparative analysis of energy and environmental impacts and evaluation of the eco-efficiency improvement potential. Building and Environment, v.46, n.5, p.1133-1140, 2011. DOI: http://doi.org/10.1016/i.buildenv.2010.12.002 
CHRISTOFOROU, E.; KYLILI, A.; FOKAIDES, P. A.; IOANNOU, I. Cradle to site Life Cycle Assessment (LCA) of adobe bricks. Journal of Cleaner Production, v.111, p.01-10, 2015. DOI: http://doi.org/10.1016/j.jclepro.2015.09.016

CORDEIRO, G. C.. Utilização de cinzas ultrafinas do bagaço de cana-de-açúcar e da casca de arroz como aditivos minerais em concreto. Tese (Doutorado em Engenharia) Universidade Federal do Rio de Janeiro, Rio de Janeiro, 2006.

DUIGOU, A.; BALEY, C.. Coupled micromechanical analysis and life cycle assessment as an integrated tool for natural fibre composites development. Journal of Cleaner

Production, v.83, p.61-69, 2014. DOI:

http://doi.org/10.1016/j.jclepro.2014.07.027

HERVY. M.; EVANGELIST, S.; LETTIERI, P.; LEE, K. Y.. Life cycle assessment of nanocellulose-reinforced advanced fibre composites. Composites Science and Technology, v.118, p.154-162, 2015. DOI: http://doi.org/10.1016/j.compscitech.2015.08.024

ILG, P.; HOEHNE, C.; GUENTHER, E.. High-performance materials in infrastructure: a review of applied life cycle costing and its drivers - the case of fiber-reinforced composites. Journal of Cleaner Production, v.112, p.926945, 2016. DOI: http://doi.org/10.1016/j.jclepro.2015.07.051

ISO. International Organization for Standardization. ISO 14040: Life cycle assessment. principles and framework. ISO, 2006.

IRIAS, L. J. M.; RODRIGUEZ, G. S; CAMPANHOLA, C.; KITAMURA, P. C.; RODRIGUES, I.; BUSCHINELLI, C. C. A. Sistema de Avaliação de Impactos Ambiental de Inovações Tecnológicas nos segmentos agropecuário, produção animal e agroindústria (SISTEMA AMBITEC). Jaguariúna: EMBRAPA Meio Ambiente, 2004.

LIMA, J. A. R.. Avaliação das consequências da produção de concreto no Brasil para as mudanças climáticas. (Doutorado em Engenharia de Construção Civil e Urbana) - Universidade de São Paulo, São Paulo, 2010.

MARQUES, V. M.. Avaliação de aspectos e impactos ambientais ao longo do ciclo de vida de telhas de fibrocimento com e sem amianto. Dissertação (Mestrado em Engenharia Civil) - Universidade do Vale do Rio dos Sinos, São Leopoldo, 2014.

RODRIGUES, G. S.; CAMPANHOLA, C.; KITAMURA, P. C.. An environmental impact assessment system for agricultural
R\&D. Environmental Impact Assessment Review, v.23, n.2, p.219-244, 2003. DOI: http://doi:10.1016/S01959255(02)00097-5

SILVA, E. J.; VELASCO, F. G.; LUZARDO, F. H. M.; MARQUES, M. L.; MILIAN, F. M.; RODRIGUES, L. B.. Compósito cimentício com elevado teor de fibra de coco tratada: propriedades físicas e durabilidade. Revista Matéria, v.23, n.3, 2018.

SILVA, E. J.; MARQUES, M. L.; VASCONCELOS, A. R. B.; AKASAKI, J. L.; TASHIMA, M. M.; PEREIRA, A. M.. Effect of milling process for rice husk ash on mechanical strength of blended Portland cement mortars. Key Engineering Materials, v.600, p.240-249, 2014. DOI: http://doi:10.4028/www.scientific.net/KEM.600.240

SILVA, R. B.; FONTES, C. M. A.; LIMA, P. R. L.; GOMES, O. F. M.; LIMA, L. G.; MOURA, R. C. A.; TOLÊDO FILHO, R. D.. Cinzas de biomassa geradas na agroindústria do cacau: caracterização e uso em substituição ao cimento. Ambiente Construído, v.15, n.4, p.321-334, 2015. DOI: http://doi.org/10.1590/s1678-86212015000400053

TEIXEIRA, L. P.; CARVALHO, F. M. A.. A construção civil como instrumento de desenvolvimento da economia brasileira. Revista paranaense de desenvolvimento, Curitiba, n.109, p.09-26, 2005

VELASCO, M. G.. Impactos ambientais e sociais da introdução de novas tecnologias do aproveitamento de resíduos de coco. Dissertação (Mestrado em Desenvolvimento regional e Meio ambiente, Universidade Estadual de Santa Cruz, Ilhéus, 2013.

VELASCO, M. G.; LUZARDO, F. H. M.; VELASCO, F. G.. Novos indicadores para a avaliação de impactos sócio ambientais da inovação tecnológica em agroindústrias de reaproveitamento de resíduos. Aplicação na agroindústria de coco. Revista Brasileira de Ciências Ambientais, n.33, p.32-44, 2014

XU, X.; JAYARAMAN, K.; MORIN, C.; PECQUEUX, N.. Life cycle assessment of wood-fibre-reinforced polypropylene composites. Journal of materials processing technology, v.98, n.1-3, p.168-177, 2008. DOI: http://doi.org/10.1016/j.jmatprotec.2007.06.087

YIN, S.; TULADHAR. R.; SHEEHAN, M.; COMBE, M.; COLLISTER, T.. A life cycle assessment of recycled polypropylene fibre in concrete footpaths. Journal of Cleaner Production, v.112, p.2231-2242, 2016. DOI: http://doi.org/10.1016/j.jclepro.2015.09.073

A CBPC - Companhia Brasileira de Produção Científica (CNPJ: 11.221.422/0001-03) detém os direitos materiais desta publicação. Os direitos referem-se à publicação do trabalho em qualquer parte do mundo, incluindo os direitos às renovações, expansões e disseminações da contribuição, bem como outros direitos subsidiários. Todos os trabalhos publicados eletronicamente poderão posteriormente ser publicados em coletâneas impressas sob coordenação da Sustenere Publishing, da Companhia Brasileira de Produção Científica e seus parceiros autorizados. Os (as) autores (as) preservam os direitos autorais, mas não têm permissão para a publicação da contribuição em outro meio, impresso ou digital, em português ou em tradução. 